\title{
Next generation mitochondrial disease: change in diagnostics with eyes on therapy
}

\author{
Eva Morava $^{1,2} \cdot$ Garry K Brown ${ }^{3}$
}

Published online: 30 April 2015

(C) SSIEM 2015

Is the paradigm of "mitochondrial disease" in 2015 different from how it was previously defined? Next generation sequencing (NGS) in patients with mitochondrial disease has revealed many novel gene defects with unexpected biochemical associations and clinical consequences whose metabolic basis remains unclear. These genes and their products are teaching us about novel metabolic concepts which are not primarily related to mitochondrial enzymes or the mitochondrial transcription, translation, or transport machineries (Honzik et al. 2015; Wortmann et al. 2015; Rahman et al. 2015; Horvath et al. 2015). Some are hypothesized to alter mitochondrial membrane integrity, affect signaling pathways, or change reactive oxygen species (ROS) production, but do not lead to severe mitochondrial oxidative phosphorylation (OXPHOS) complex deficiencies. In most of the recently discovered mitochondrial disorders, classical enzyme histochemistry in biopsied muscle would probably not support a diagnosis of primary mitochondrial dysfunction (Wortmann et al. 2015). Other markers of global mitochondrial dysfunction, such as oxygen consumption, ATP production, and ROS production, are frequently abnormal in these novel mitochondrial disorders. However, these changes are relatively non-specific,

Communicated by: Verena Peters

Eva Morava

emoravakozicz@TULANE.EDU

1 Department of Pediatrics, Tulane University Medical School, New Orleans, LA, USA

2 Department of Pediatrics, University Hospital Leuven, Leuven, Belgium

3 Oxford Medical Genetics Laboratories, The Churchill Hospital, Oxford University Hospitals NHS Trust, Oxford, UK and including them as part of routine diagnostic work-up may not improve the success in identifying particular conditions.

We need to reconsider whether we can rely on the current practice of measuring classical biochemical changes, such as OXPHOS complex deficiencies, to establish a diagnosis of mitochondrial disease. Instead of questioning whether these novel gene defects are "real" mitochondrial disorders, we suggest reevaluating our definitions and diagnostic strategies. With the broad availability of NGS, we should focus on noninvasive diagnostics in suspected mitochondrial disease. It is time to update our diagnostic flowchart (Fig. 1).

Naturally, the clinical work-up remains the same; start with the mitochondrial disease score by looking for muscle, central nervous system (CNS), and multisystem symptoms; evaluate for biochemical abnormalities in blood and urine; and use CNS imaging (Morava et al. 2006). The next step however is not a muscle biopsy, but mitochondrial DNA (mtDNA) analysis (deletion studies and sequencing) (Rahman et al. 2015). If this is normal, then we should proceed to NGS, either using nuclear gene panels or performing whole exome sequencing. This strategy would also allow us to diagnose secondary mitochondrial diseases due to muscle disorders or syndromes (Wortmann et al. 2015). If the results are ambiguous, one can still consider muscle, liver, or skin biopsy to obtain more reliable data about mtDNA deletion/depletion or a biochemical diagnosis.

How are paradigm changes in treatment affecting the longterm disease outcome? Here, we expect to see even bigger changes in the future. In past decades, we aimed at preserving or improving mitochondrial function. Treatment strategies were mostly aimed at correcting measured biochemical changes or trying to regulate ROS production. Our primary focus was on supportive therapy to obtain the best quality of life, and the goal of a cure seemed a long way off. Now, with the new focus on gene therapy, such a goal may be coming 
Diagnostic flow chart for mitochondrial disease

1. Phenotype analysis including laboratory evaluations in blood and urine

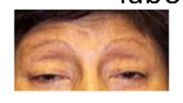

and brain imaging

$\downarrow$

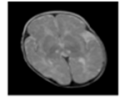

2. Mitochondrial disease score

3. mtDNA analysis and consider nuclear DNA panels

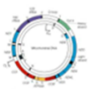

$\downarrow$

4. Next generation sequencing
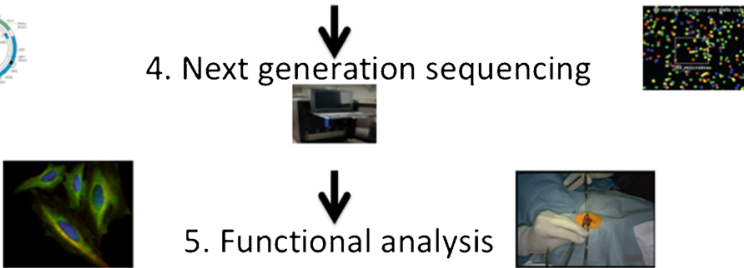

5. Functional analysis

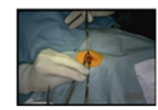

Fig. 1 Diagnostic flow chart for mitochondrial disease

closer. However, we will only be able to exploit fully all of the new treatment possibilities if we are also able to make the widest use of the new diagnostic tests to define underlying genetic defects in the "new" mitochondrial diseases rapidly and precisely.

Conflict of interest None.

\section{References}

Honzik T (2015) TMEM70 deficiency: long-term outcome of 48 patients and suggested management recommendations. J Inherit Metab Dis

Horvath R (2015) Reversible infantile mitochondrial diseases. J Inherit Metab Dis

Wortmann S (2015) Whole exome sequencing as a routine diagnostic test for mitochondrial disorders. J Inherit Metab Dis

Rahman S (2015) Paediatric single mitochondrial DNA deletion disorders: an overlapping spectrum of disease. J Inherit Metab Dis

Morava E, van den Heuvel L, Hol F, de Vries MC, Hogeveen M, Rodenburg RJ, Smeitink JA (2006) Mitochondrial disease criteria: diagnostic applications in children. Neurology 67:1823-6 